\title{
On a one-dimensional analogue of the Smale horseshoe
}

\author{
by RYsZARD RudNiCKI (Katowice)
}

Abstract. We construct a transformation $T:[0,1] \rightarrow[0,1]$ having the following properties:

1) $(T,|\cdot|)$ is completely mixing, where $|\cdot|$ is Lebesgue measure,

2) for every $f \in L^{1}$ with $\int f d x=1$ and $\varphi \in C[0,1]$ we have $\int \varphi\left(T^{n} x\right) f(x) d x \rightarrow$ $\int \varphi d \mu$, where $\mu$ is the cylinder measure on the standard Cantor set,

3) if $\varphi \in C[0,1]$ then $n^{-1} \sum_{i=0}^{n-1} \varphi\left(T^{i} x\right) \rightarrow \int \varphi d \mu$ for Lebesgue-a.e. $x$.

1. Introduction. Let $T$ be a measurable transformation of a given measure space $(X, \Sigma, m)$. One of the ways of describing the chaotic behaviour of this transformation is to prove the existence of an invariant measure $\mu \ll m$ having some mixing properties. But in some important cases such as the Hénon map or generalized Lozi maps there are no invariant measures absolutely continuous with respect to the given (Lebesgue) measure $m$. In these cases the chaos can be identified with the existence of strange attractors or Bowen-Ruelle measures [2, 3]. For example L.-S. Young [7] proved that the generalized Lozi maps have Bowen-Ruelle measures. A more unified approach to the notion of chaos was proposed by Lasota [4]. Namely, a non-singular transformation of a compact topological space $X$ with a given Borel measure $m$ is chaotic if there is a non-trivial probability measure $\mu$ such that

$$
\int \varphi\left(T^{n} x\right) f(x) m(d x) \rightarrow \int \varphi(x) \mu(d x) \int f(x) m(d x)
$$

for every $\varphi \in C(X)$ and $f \in L^{1}(X, m)$. The last condition means that if $\nu$ is a probability measure and $\nu \ll m$ then $\nu \circ T^{-n}$ converges weak ${ }^{*}$ to some fixed limit measure $\mu$.

In this paper we give an example of a chaotic transformation of the unit interval whose limit measure is the cylinder measure on the standard 
Cantor set. This transformation has some additional properties, namely, $T$ is completely mixing and the limit measure $\mu$ is a Bowen-Ruelle measure. Our transformation exhibits dynamics akin to that of the Smale horseshoe on horizontal and vertical segments, e.g. $T^{n}(x)$ converges a.e. to the Cantor set $C, T$ is a Bernoulli endomorphism of $C$, and $T$ is piecewise expanding.

2. Stochastic attractor. Let $X$ be a compact metric space and let $\mathcal{B}(X)$ denote the family of all Borel subsets of $X$. A probability Borel measure $\mu$ is called non-trivial if $\operatorname{supp} \mu$ contains at least two points. Let $m$ be a given $\sigma$-finite Borel measure on $X$. Denote by $D$ the subspace of $L^{1}(X, \mathcal{B}(X), m)$ containing all non-negative functions $f$ with $\int f d m=1$. Let $T: X \rightarrow X$ be a non-singular transformation with respect to $m$ and let $P: D \rightarrow D$ be the Frobenius-Perron operator corresponding to $T$, i.e.

$$
\int_{A} P f d m=\int_{T^{-1}(A)} f d m
$$

for every $A \in \mathcal{B}(X)$ and $f \in D$.

The transformation $T$ is called chaotic if there exists a non-trivial measure $\mu$ such that

$$
\lim _{n} \int \varphi(x) P^{n} f d x=\int \varphi(x) d \mu
$$

for every $\varphi \in C(X)$ and $f \in D$. Such a measure $\mu$ will be called the limit measure. It is easy to verify that the limit measure is invariant under $T$. Condition (2.1) is equivalent to (1.1).

The system $(X, \mathcal{B}(X), m ; T)$ is called completely mixing if for every $f \in L^{1}$ with $\int f d m=0$ we have

$$
\lim _{n}\left\|P^{n} f\right\|=0
$$

where $\|\cdot\|$ is the norm in $L^{1}(X, m)$. The system $(X, \mathcal{B}(X), m ; T)$ is completely mixing iff the $\sigma$-algebra $\bigcap_{n=1}^{\infty} T^{-n}(\mathcal{B}(X))$ contains only sets of measure zero or one [6].

Let $T$ be chaotic with limit measure $\mu, Y=\operatorname{supp} \mu$ and assume that the system $(X, \mathcal{B}(X), m ; T)$ is completely mixing. Then the pair $(Y, \mu)$ will be called a stochastic attractor for $(X, \mathcal{B}(X), m ; T)$.

Now denote by $|\cdot|$ Lebesgue measure on the interval $I=[0,1]$. The measure space $(I, \mathcal{B}(I),|\cdot|)$ is isomorphic to $(X, \mathcal{B}(X), m)$, where $X=\{0,1,2\}^{N}$ and $m$ is the product of measures $m_{i}$ on $\{0,1,2\}$ such that $m_{i}(\{j\})=\frac{1}{3}$ for $j=0,1,2$. The isomorphism $i: X \rightarrow I$ is given by the formula $i(x)=\sum_{k=1}^{\infty} x_{k} / 3^{k}$. It induces a metric $\rho$ on $X$ by $\rho(x, y)=|i(x)-i(y)|$. We define a map $S: X \rightarrow X$ by $S\left(\left(x_{1}, x_{2}, \ldots\right)\right)=\left(x_{2}, \ldots, x_{n-1}, x_{n+1}, \ldots\right)$ if $1 \notin\left\{x_{1}, \ldots, x_{n-1}\right\}$ and $x_{n}=1$ or if $x_{1}=\ldots=x_{n-1}=1$ and $x_{n} \neq 1$, 
$n \geq 2$. In other words, the sequence $S(x)$ is obtained from $x$ by removing the first 1 and the first $x_{i}$ different from 1 . If $x$ does not contain 1's then we put $S(x)=\left(x_{2}, x_{3}, \ldots\right)$. Now we can define a transformation $T: I \rightarrow I$ by $T(i(x))=i(S(x))$ for $x \in X$. It is easy to check that $T$ is piecewise linear, more exactly, there are a countable number of open intervals $I_{1}, I_{2}, \ldots$ such that $T$ is linear on $I_{k}, k=1,2, \ldots, T^{\prime}(x)=9$ for $x \in \bigcup I_{k}$ and $\left|\bigcup I_{k}\right|=1$. Moreover, $T$ is discontinuous on a countable set, which implies that $T$ is a measurable transformation. From the above facts it follows that $T$ is a non-singular transformation, i.e. $\left|T^{-1}(A)\right|=0$ if $|A|=0$.

We need some additional notation. We put

$$
\begin{aligned}
C_{i_{1} \ldots i_{n}} & =\left\{x \in X: x_{1}=i_{1}, \ldots, x_{n}=i_{n}\right\}, \\
C_{n} & =\bigcup\left\{C_{i_{1} \ldots i_{n}}:\left(i_{1}, \ldots, i_{n}\right) \in\{0,2\}^{n}\right\}, \\
C & =\bigcap_{n=1}^{\infty} C_{n} .
\end{aligned}
$$

Then $i(C)$ is the standart Cantor set. Let $\nu$ be the product of the measures $\nu_{n}$ on $\{0,1,2\}$ such that $\nu_{n}(\{0\})=\nu_{n}(\{2\})=\frac{1}{2}$ and $\nu_{n}(\{1\})=0$ for $n=$ $1,2, \ldots$ By $\mu$ we denote the Borel measure on $I$ given by $\mu(A)=\nu\left(i^{-1}(A)\right)$ for $A \in \mathcal{B}(I)$.

THEOREM 1. The pair $(i(C), \mu)$ is a stochastic attractor for the system $(I, \mathcal{B}(I),|\cdot| ; T)$. Moreover, the measure $\mu$ is a Bowen-Ruelle measure, i.e. for $\varphi \in C(I)$ we have

$$
n^{-1} \sum_{k=0}^{n-1} \varphi\left(T^{k} x\right) \rightarrow \int \varphi d \mu
$$

for Lebesgue-a.e. $x$.

3. Proof of Theorem 1. Since the systems $(I, \mathcal{B}(I),|\cdot| ; T)$ and $(X, \mathcal{B}(X), m ; S)$ are isomorphic and $\mu=\nu \circ i^{-1}$ it is sufficient to prove the analogous theorem for the second system and the measure $\nu$. We split the proof into six lemmas.

LEMMA 1. Let $N_{p}(x)$ denote the number of 1 's in the sequence $\left(x_{1}, \ldots, x_{p}\right)$. Then for $m$-a.e. $x$ there exists $k(x, p)$ such that $N_{p}\left(S^{k} x\right)=0$ for $k \geq k(x, p)$.

Proof. Since $m$ is a product measure of identical measures, the strong law of large numbers implies $N_{n}(x) / n \rightarrow \frac{1}{3}$ for $m$-a.e. $x$. Let $n_{0}(x)$ be an integer such that $N_{n}(x)<\frac{2}{5} n$ for $n \geq n_{0}(x)$. We put $k(x, p)=\max \left\{2 p, n_{0}\right\}$. Then for $k \geq k(x, p)$ the sequence $\left(x_{1}, \ldots, x_{2 k+p}\right)$ contains at most $k 1$ 's. 
Indeed,

$$
N_{2 k+p}(x)<\frac{2}{5}(2 k+p) \leq k .
$$

Since $S^{k} x$ is obtained from $x$ by removal of the first $k 1$ 's and the first $k$ terms different from 1 , the first $p$ terms of $S^{k} x$ are different from 1 .

Corollary 1. If $k \geq k(x, p)$ then $S^{k} x \in C_{p}$.

Corollary 2. $\lim _{n \rightarrow \infty} \rho\left(S^{n} x, C\right)=0$.

Lemma 2. For every $p$ and $f \in D$ we have

$$
\lim _{n \rightarrow \infty} \int_{C_{p}} P^{n} f d m=1 .
$$

Proof. Since

$$
\int_{C_{p}} P^{n} f d m=\int_{S^{-n}\left(C_{p}\right)} f d x
$$

it is sufficient to prove that $\lim _{n} m\left(S^{-n}\left(C_{p}\right)\right)=1$. We set $A_{n}=S^{-n}\left(C_{p}\right)$, $A_{n, \infty}=\bigcap_{k=n}^{\infty} A_{k}$ and $B=\bigcup_{n=1}^{\infty} A_{n, \infty}$. Then from the definition of $B$ it follows that $x \in B$ iff there exists $k_{0}(x)$ such that $S^{k} x \in C_{p}$ for $k \geq k_{0}(x)$. This implies that $m(B)=1$ and consequently $\lim _{n} m\left(A_{n}\right)=1$.

Lemma 3. Let $\left(x_{1}, \ldots, x_{n}\right) \in\{0,2\}^{n}$. Then

$$
\lim _{k \rightarrow \infty} m\left(S^{-k}\left(C_{x_{1} \ldots x_{n}}\right)\right)=2^{-n} .
$$

Proof. Let $\left(x_{1}, \ldots, x_{n}\right) \in\{0,2\}^{n}$ and $\left(y_{1}, \ldots, y_{n}\right) \in\{0,2\}^{n}$. From the definition of the measure $m$ and the transformation $S$ it follows that

$$
m\left(S^{-k}\left(C_{x_{1} \ldots x_{n}}\right)\right)=m\left(S^{-k}\left(C_{y_{1} \ldots y_{n}}\right)\right) .
$$

Hence $m\left(S^{-k}\left(C_{x_{1} \ldots x_{n}}\right)\right)=2^{-n} m\left(S^{-k}\left(C_{n}\right)\right)$. This and Lemma 2 imply (3.1).

LEMMA 4. If $h \in C(X)$ then

$$
\lim _{k \rightarrow \infty} \int_{X} h P^{k} 1 d m=\int_{X} h d \nu .
$$

Proof. Fix $\varepsilon>0$. Since $h$ is uniformly continuous there exists a $\delta>0$ such that $\rho(x, y)<\delta$ implies $|h(x)-h(y)|<\varepsilon$. Let $n$ be a positive integer such that $3^{-n}<\delta$. We define a new function $\bar{h}: X \rightarrow \mathbb{R}$ by setting $\bar{h}(x)=h\left(x_{1}, \ldots, x_{n}, 0,0, \ldots\right)$. The function $\bar{h}$ is constant on each set $C_{x_{1} \ldots x_{n}}$ and $|h(x)-\bar{h}(x)|<\varepsilon$. From Lemma 3 we obtain

$$
\lim _{k \rightarrow \infty} \int_{C_{n}} \bar{h} P^{k} 1 d m=\int_{C_{n}} \bar{h} d \nu=\int_{X} \bar{h} d \nu .
$$


From the inequality $|h(x)-\bar{h}(x)|<\varepsilon$ it follows that

$$
\left|\int_{C_{n}} h P^{k} 1 d m-\int_{X} h d \nu\right| \leq 2 \varepsilon+\left|\int_{C_{n}} \bar{h} P^{k} 1 d m-\int_{X} \bar{h} d \nu\right| .
$$

This and (3.3) imply

$$
\limsup _{k \rightarrow \infty}\left|\int_{C_{n}} h P^{k} 1 d m-\int_{X} h d \nu\right| \leq 2 \varepsilon .
$$

According to Lemma 2 we have

$$
\lim _{k \rightarrow \infty} \int_{X \backslash C_{n}} h P^{k} 1 d m=0
$$

and consequently

$$
\limsup _{k \rightarrow \infty}\left|\int_{X} h P^{k} 1 d m-\int_{X} h d \nu\right| \leq 2 \varepsilon,
$$

which implies (3.2).

LEMMA 5. A pair $(C, \nu)$ is a stochastic attractor for the system $(X, \mathcal{B}(X), m ; S)$.

In the proof of Lemma 5 we will use the following Hewitt-Savage theorem (see [1], Section 6).

THEOREM 2. Let $X_{1}, X_{2}, \ldots$ be a sequence of independent identically distributed random variables. Denote by $\mathcal{A}_{n}$ the $\sigma$-algebra generated by the sets of the form

$$
A=\left\{\left(X_{1}, \ldots, X_{n}\right) \in M\right\} \cap\left\{X_{n+1} \in H_{1}\right\} \cap \ldots \cap\left\{X_{n+j} \in H_{j}\right\}
$$

where $j$ is a positive integer, $H_{1}, \ldots, H_{j} \in \mathcal{B}\left(\mathbb{R}^{1}\right)$ and $M \in \mathcal{B}\left(\mathbb{R}^{n}\right)$ is a symmetric set, i.e. if $\left(x_{1}, \ldots, x_{n}\right) \in M$ and $\pi$ is any permutation of $\{1, \ldots, n\}$ then also $\left(x_{\pi(1)}, \ldots, x_{\pi(n)}\right) \in M$. Then the $\sigma$-algebra $\bigcap_{n=1}^{\infty} \mathcal{A}_{n}$ contains only sets of measure 0 or 1 .

Proof of Lemma 5. First we check that if $A \in \bigcap_{n=1}^{\infty} T^{-n}(\mathcal{B}(X))$ then $m(A)=0$ or $m(A)=1$. The set $A$ has the following property: if $x \in A$ and for some integer $n$ we have $T^{n} x=T^{n} y$ then $y \in A$. Now assume that $x \in A, y \in X, N_{n}(x)=N_{n}(y)$ for some integer $n$, and $x_{k}=y_{k}$ for $k \geq n+1$. Then $T^{n} x=T^{n} y$ and consequently $y \in A$. Now we can use the Hewitt-Savage theorem. In our case $X_{i}$ denotes the $i$ th term of $x$. Since no permutation of $\left\{x_{1}, \ldots, x_{n}\right\}$ changes the number of 1 's, we have $A \in \mathcal{A}_{n}$ for $n \geq 1$. Therefore $A \in \bigcap_{n=1}^{\infty} \mathcal{A}_{n}$ and consequently $m(A)=0$ or $m(A)=1$. This implies that $T$ is completely mixing. Now we take $f \in D$. Then putting $g=f-1$ we obtain $\left\|P^{n} g\right\| \rightarrow 0$, which implies that for every $h \in C(X)$ we have $\int h P^{n} g d m \rightarrow 0$. This and Lemma 4 give $\int h P^{n} f d m \rightarrow \int h d \nu$. 
LEMma 6. $\nu$ is a Bowen-Ruelle measure.

Proof. We define a function $\Phi: X \rightarrow \mathbb{C}$ by assuming that $\Phi(x)$ is the sequence obtained from $x=\left(x_{1}, x_{2}, \ldots\right)$ by removal of all terms equal to 1 . From Lemma 1 it follows that $\rho\left(S^{n} x, S^{n} \Phi(x)\right) \rightarrow 0$ as $n \rightarrow \infty$. This implies that for every $h \in C(X)$

$$
\lim _{n \rightarrow \infty} n^{-1} \sum_{k=0}^{n-1}\left[h\left(S^{k} x\right)-h\left(S^{k} \Phi(x)\right)\right]=0 .
$$

Since $S$ is a Bernoulli shift on $C$ and $\nu$ is an invariant measure the individual ergodic theorem implies

$$
\lim _{n \rightarrow \infty} n^{-1} \sum_{k=0}^{n-1} h\left(S^{k} y\right)=\int h d \nu
$$

for $\nu$-a.e. $y$. In order to complete the proof it is sufficient to show that

$$
\nu(A)=m\left(\Phi^{-1}(A)\right) \quad \text { for } A \in \mathcal{B}(X) .
$$

First we check (3.5) for $A=C_{x_{1} \ldots x_{n}}$, where $\left(x_{1}, \ldots, x_{n}\right) \in\{0,2\}^{n}$. Let $B=C_{y_{1} \ldots y_{n}}$ be another cylinder subset of $C_{n}$. Then $\Phi^{-1}(A)$ and $\Phi^{-1}(B)$

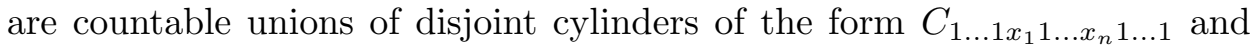
$C_{1 \ldots 1 y_{1} 1 \ldots y_{n} 1 \ldots 1}$ respectively. From the definition of the measure $m$ it follows that $m\left(\Phi^{-1}(A)\right)=m\left(\Phi^{-1}(B)\right)$ and consequently $m\left(\Phi^{-1}(A)\right)=\nu(A)$. If $A=C_{x_{1} \ldots x_{n}}$ and $A$ is disjoint from $C_{n}$ then $\nu(A)=m\left(\Phi^{-1}(A)\right)=0$. This implies that for each cylinder set $A$ we have $m\left(\Phi^{-1}(A)\right)=\nu(A)$ and consequently (3.5) holds.

Rem arks. 1. In [5] A. Lasota and J. A. Yorke considered piecewise $C^{2}$ transformations $\tau: I \rightarrow I$ with a finite number of "pieces". They proved that if inf $\left|\tau^{\prime}\right|>1$ then $\tau$ has an invariant probability measure absolutely continuous w.r.t. Lebesgue measure. Our transformation shows that the theorem of Lasota and Yorke cannot be extended to a "countable" piecewise transformation. Indeed, from condition (2.1) it follows that the operator $P$ has no fixed points in the set $D$, which implies that $T$ has no invariant measures $\mu \ll|\cdot|$.

2. In the paper we consider three properties of transformations: chaos, complete mixing, existence of a Bowen-Ruelle measure. There are some open problems concerning relations between these properties. In particular, the following two questions are interesting:

a) Let $(X, \mathcal{B}(X), m ; T)$ be a completely mixing system on a compact metric space $X$. Does there exist a measure $\mu$ satisfying condition (1.1)? We do not assume that $\mu$ is non-trivial. 
b) Let $(\operatorname{supp} \mu, \mu)$ be a stochastic attractor for the system $(X, \mathcal{B}(X), m ; T)$. Is $\mu$ a Bowen-Ruelle measure?

\section{References}

[1] P. Billingsley, Probability and Measure, Wiley, New York 1979.

[2] R. Bowen, Equilibrium States and the Ergodic Theory of Anosov Diffeomorphisms, Lecture Notes in Math. 47, Springer, Berlin 1975.

[3] R. Bowen and D. Ruelle, The ergodic theory of Axiom A flows, Invent. Math. 29 (1975), 181-202.

[4] A. Lasota, Thoughts and conjectures on chaos, preprint.

[5] A. Lasota and J. A. Yorke, On the existence of invariant measures for piecewise monotonic transformations, Trans. Amer. Math. Soc. 186 (1973), 481-488.

[6] M. Lin, Mixing for Markov operators, Z. Wahrsch. Verw. Gebiete 19 (1971), 231-242.

[7] L.-S. Young, Bowen-Ruelle measures for certain piecewise hyperbolic maps, Trans. Amer. Math. Soc. 287 (1985), 41-48.

INSTITUTE OF MATHEMATICS

SILESIAN UNIVERSITY

BANKOWA 14

40-007 KATOWICE, POLAND 